La Revue

des Droits

de l'Homme

\section{La Revue des droits de l'homme}

Revue du Centre de recherches et d'études sur les droits fondamentaux

$6 \mid 2014$

Revue des droits de l'homme $-\mathrm{N}^{\circ} 5$

\title{
La première décision au fond de la Cour africaine des droits de l'homme et des peuples
}

(Arrêt du 14 juin 2013 sur les affaires jointes Tangayika Law Society \& The Legal and Human Rights Centre c. Tanzanie et Révérend Christopher R. Mtikila c. Tanzanie)

\section{Alain Didier Olinga}

\section{(2) OpenEdition}

\section{Journals}

Electronic version

URL: http://journals.openedition.org/revdh/953

DOI: $10.4000 /$ revdh.953

ISSN: 2264-119X

\section{Publisher}

Centre de recherches et d'études sur les droits fondamentaux

\section{Electronic reference}

Alain Didier Olinga, « La première décision au fond de la Cour africaine des droits de l'homme et des peuples », La Revue des droits de l'homme [Online], 6 | 2014, Online since 16 November 2014, connection on 08 July 2020. URL : http://journals.openedition.org/revdh/953 ; DOI : https://doi.org/ 10.4000/revdh.953

This text was automatically generated on 8 July 2020 .

Tous droits réservés 


\section{La première décision au fond de la Cour africaine des droits de l'homme et des peuples}

(Arrêt du 14 juin 2013 sur les affaires jointes Tangayika Law Society \& The Legal and Human Rights Centre c. Tanzanie et Révérend Christopher R. Mtikila c. Tanzanie)

\section{Alain Didier Olinga}

1 La première décision au fond de la Cour africaine des droits de l'homme et des peuples était attendue par l'ensemble de ceux qui s'intéressent au fonctionnement et au devenir $\mathrm{du}$ système africain de garantie des droits fondamentaux ${ }^{1}$. Après de nombreuses décisions d'incompétence ${ }^{2}$, l'occasion lui en a été offerte par le groupe d'affaires n 009/2011 et 011/2011 contre la République Unie de Tanzanie, l'un des rares Etats à avoir souscrit la déclaration de l'article 36 paragraphe 4 du Protocole de Ouagadougou et à être ainsi exposé au risque d'être attrait devant la Cour par des ONG et des individus ${ }^{3}$. On ne peut que saluer ce premier pas, car il permet de voir comment la Cour aborde des questions de fond du droit, ou de droit substantiel, et comment sur ces questions elle se positionne par rapport à l'acquis jurisprudentiel de la Commission de Banjul. Pour autant, il n'est pas sûr que cette première sortie au fond laissera un souvenir indélébile; en effet, à notre avis, elle laisse interrogateur sur un certain nombre d'aspects, au point de créer une situation d'incertitude sur des aspects processuels et de fond dans le système de protection articulé autour de la Charte africaine des droits de l'homme et des peuples. Avant de les relever, il importe naturellement de revenir un tant soit peu sur les faits de la cause.

Les données factuelles qui ont donné lieu à l'arrêt du 14 juin 2013 sont simples. En 1992, est adoptée en Tanzanie une révision constitutionnelle prescrivant l'affiliation à un parti politique de tout candidat potentiel aux élections présidentielles, parlementaires et locales et excluant, de jure et de facto, toute candidature indépendante ${ }^{4}$. Cette révision sera contestée devant les instances judiciaires par M. Mtikila, avec succès, puisque en octobre 1994 la Haute Cour lui donne raison. En décembre 1994, l'Assemblée nationale 
adopte un projet de loi réaffirmant l'interdiction des candidatures indépendantes, loi entrée en vigueur en janvier 1995. M. Mtikila conteste à nouveau cette loi devant la Haute Cour en 2005, soit dix ans après son entrée en vigueur, avec succès, car la Haute Cour lui donne une fois de plus raison. Cette décision de la Haute Cour est contestée en 2009 par l'Attorney General devant la Cour d'Appel, laquelle, dans un arrêt du 17 juin 2010, infirme la position de la Haute Cour et confirme l'interdiction des candidatures indépendantes. Il s'agit ainsi d'un processus judiciaire interne assez saccadé, étalé sur près de vingt ans. C'est suite à cette décision de la Cour d'Appel que deux requêtes vont être déposées devant la Cour africaine les 2 et 10 juin par les premier et second requérants, à l'effet de faire constater que l'interdiction des candidatures indépendantes méconnaît le droit de participer à la direction des affaires publiques de son pays, la liberté d'association, le droit à l'égalité et à la non discrimination et le principe de l'Etat de droit. La Cour, à l'unanimité, après un peu plus de deux ans de procédure (du 2 juin 2011 au 16 juin 2013) va constater la violation par la Tanzanie des deux premiers droits ; à la majorité de sept contre deux, elle constate la violation du troisième droit, mais les deux juges dissidents n'ont pas formulé, comme ils en ont le droit sans y être pour autant astreints, d'opinion dissidente explicitant leur position. Elle estime que la question de la violation de l'Etat de droit est sans intérêt. La Cour ordonne à la Tanzanie de " prendre toutes les mesures constitutionnelles, législatives et autres dispositions utiles dans un délai raisonnable, afin de mettre fin aux violations constatées et informer la Cour des mesures prises à cet égard ». Elle réserve la question de la réparation à un examen ultérieur, le temps que M. Mtikila précise ses exigences à cet égard.

3 La décision concerne ainsi une question constitutionnelle de la plus haute importance et de grande sensibilité pour de nombreux Etats africains. D'ailleurs, le défendeur a fait valoir que «la question des candidatures indépendantes est une question politique et non juridique» (paragraphe 94 de l'arrêt). Cette question a déjà été abordée par d'autres mécanismes internationaux de contrôle, notamment le Comité des droits de l'homme $e^{5}$ et la Cour interaméricaine des droits de l'homme ${ }^{6}$. Pour mieux apprécier les enseignements que l'on peut tirer de cette première décision au fond, il semble indiqué d'aborder successivement les questions de procédure, dont le traitement apparaît prometteur, puis les aspects de fond proprement dits, dont l'examen parait plus discutable et moins convaincant.

\section{Un traitement prometteur des questions de procédure par la Cour}

4 La procédure est un aspect essentiel du contentieux, et particulièrement du contentieux international ${ }^{7}$. Elle est un élément qui légitime le travail technique du juge, et prépare ce faisant l'acceptation de ses décisions. Dans cette affaire, l'on a observé une continuité dans la rigueur avec laquelle est appliqué le principe de récusation des juges, avec l'abstention à siéger du juge Ramadani, de nationalité tanzanienne ${ }^{8}$. La jonction des requêtes a paru plus hésitante. Au paragraphe 78, elle est justifiée du fait de "positions sensiblement similaires » des deux requérants, alors qu'au paragraphe 89.1, ces positions sont "essentiellement identiques ", même si la Cour se réserve de traiter différemment les deux requêtes quand cela serait nécessaire. De telles formules ne sont pas sans susciter quelque perplexité quant à la pratique de la jonction des requêtes devant la Cour. Au-delà de ces aspects, trois questions méritent un examen 
particulier sur le terrain de la procédure : la question du locus standi des requérants, l'examen des conditions de recevabilité par la Cour et la question de la compétence ratione temporis de la Cour.

\section{A. La question du locus standi des requérants} Protocole de Ouagadougou, et que les ONG auteurs du premier recours avaient bien le statut d'observateurs auprès de la Commission de Banjul. De ce point de vue, tous les requérants avaient incontestablement qualité pour agir. Mais cette qualité est potentiellement la même pour tous ceux qui se trouvent dans cette situation, à l'égard d'un Etat ayant souscrit la déclaration de l'article 34(6). Cela leur confère-t-il, pour autant, en toutes situations de violations alléguées des droits, le locus standi ? La Cour ne s'est pas spécialement intéressée à ce point, probablement parce que le défendeur luimême n'a pas eu à cet égard une attitude particulièrement offensive et combative. Seul le juge Ouguergouz, dans son opinion individuelle, a invité la Cour à se montrer à l'avenir plus vigilante sur la question de l'intérêt pour agir des requérants. Au paragraphe 26 de son opinion, il estime qu' " une action devant la Cour n'est en effet recevable que si son auteur justifie de son intérêt propre à l'engager. Pour faire la preuve de cet intérêt, le requérant doit en conséquence démontrer que l'action ou l'abstention de l'Etat défendeur concerne un droit dont ledit requérant est titulaire ou le droit d'un individu au nom duquel le requérant souhaite intervenir ». En réalité, l'auteur de la requête doit démontrer en quoi il est "victime » de ce qu'il impute à l'Etat comme fait illicite au regard de la Charte, ou en quoi ceux au nom desquels il agit sont «victimes" de la violation alléguée. De manière plus générale, la Cour doit clarifier le point de savoir si le droit de recours actionné devant elle par des individus ou des ONG est un droit de recours potentiellement objectif, une démarche d'actio popularis. Cette question n'est pas banale, car les Etats encore hésitants à entrer dans le jeu de la déclaration de l'article 34(6) pourraient se montrer encore plus réticents à l'intégrer, si le prétoire de la Cour devait être encombré de requêtes à propos desquelles les plaignants n'auraient pas besoin de montrer en quoi ce qu'ils imputent à l'Etat leur a causé un préjudice ; une telle situation pourrait transformer le prétoire de la Cour en une tribune de contestation générale des évolutions politiques et sociales au sein des Etats parties à la Charte. leurs membres, membres dont l'identité n'est pas connue et dont il n'est établi à aucun moment que l'application des dispositions constitutionnelles querellées leur a causé le moindre préjudice et, le second, pour son propre compte. Là encore, si ce dernier, responsable de parti ayant pris part aux élections dans son pays sans succès, a contesté au niveau des juridictions nationales l'exclusion des candidatures indépendantes, il n'établit pas en quoi cette exclusion lui a concrètement causé un préjudice. Dans les deux cas, le dommage causé ou susceptible de l'être à des individus concrets, est vague. L'on est donc en plein contentieux objectif, et l'on peut se demander si la Cour, désireuse sans doute d'avoir enfin une décision au fond et entrainée par la communauté des ONG, n'a pas ouvert une voie qu'elle pourrait avoir du mal à refermer, même si ce faisant elle rejoint une approche déjà essayée au niveau de la Commission' 9 Il serait judicieux pour la Cour, dans le respect de son autonomie, de s'inspirer de la démarche de son homologue européenne. Devant la Cour de Strasbourg en effet, une 
ONG ne peut introduire un recours et se prétendre victime simplement parce qu'elle a pour mandat, par ses statuts, de promouvoir une cause relative aux droits de l'homme. Elle doit être victime, elle-même, d'un acte ou d'une omission qui viole la Convention européenne des droits de l'homme. De même, pour ce qui est de l'individu, sous réserve des situations de victime indirecte ou de victime potentielle, il doit avoir un intérêt personnel à agir et ne peut demander un examen in abstracto de la conformité d'un texte national à l'instrument international. Le comportement querellé de l'Etat ne doit pas seulement "concerner un droit», selon les termes du juge Ouguergouz, mais lui porter atteinte de manière spécifique et concrète ${ }^{10}$.

7 En tout état de cause, comme il n'y avait pas de dommage individualisé, toute la discussion judiciaire est restée pour beaucoup un exercice théorique, un discours principiel. Or, l'on peut douter de ce que la fonction d'une instance judiciaire soit d'effectuer des exercices théoriques de ce type, plutôt que de trancher, dans des causes bien précises, des problèmes mettant en jeu des droits concrets et effectifs.

Deux affirmations de la Cour sont à cet égard problématiques à notre avis. Au paragraphe 110 in fine, elle affirme qu' " une affaire comme celle en l'espèce ne peut et ne doit pas être examinée comme s'il s'agissait d'une action personnelle et il serait dangereux pour la Cour de donner cette impression. S'il y a violation, elle affecte tous les Tanzaniens; et si la Cour fait droit à la requête introduite par le Requérant, cette décision profitera à tous les Tanzaniens ». Au paragraphe 111, elle conclut en affirmant que «les Tanzaniens ne sont donc pas libres de participer à la direction des affaires publiques de leur pays, directement ou par le libre choix de leurs représentants ». Ces deux affirmations laissent réellement songeur. Si le droit de recours ouvert aux individus et ONG est une déclinaison du recours individuel, il est clair que les actions introduites, à défaut d'être personnelles, doivent être personnalisées, individualisées, cibler des atteintes portées à des droits dans le chef d'individus concrets et identifiables. Le recours individuel n'est pas un recours dans l'intérêt direct et objectif du droit des droits de l'homme, mais d'abord dans l'intérêt de droits subjectifs d'individus concrets. Or, il n'y avait pas devant la Cour un requérant ou une victime nommé (e) «les Tanzaniens ». De plus, les Tanzaniens n'ont mandaté ni les deux ONG, ni M. Mtikila pour défendre leurs droits à Arusha. Il n'est même pas démontré dans l'arrêt que, au-delà des actions des requérants, en Tanzanie puis devant la Cour, la question objet des requêtes agitait particulièrement la société tanzanienne et que les Tanzaniens la considéraient comme une question cruciale et vitale pour l'exercice de leurs droits politiques ${ }^{11}$. Affirmer, sur la seule base de l'exclusion des candidatures indépendantes, que «les Tanzaniens ne sont pas libres de participer à la direction des affaires publiques de leur pays " paraît si exagéré que l'on se demande comment la Cour a pu réunir l'unanimité sur une telle conclusion.

Si la Cour estime qu'il est «dangereux " pour elle de donner l'impression que l'action judiciaire de M. Mtikila est une action personnelle, individuelle, il faudrait déterminer sur quel fondement l'on va octroyer une réparation à ce deuxième requérant. Le paiement d'une juste compensation ou l'octroi d'une réparation aurait pour objet de réparer exactement quel préjudice, quel dommage personnellement subi? Cette question est importante car, de bout en bout de l'arrêt, il n'est fait nulle part mention d'un dommage matériel subi par le deuxième requérant intuitu personae, sauf l'allusion faite au paragraphe 110 au «fardeau que représente la création et l'entretien d'un parti politique», fardeau non quantifié exprimé par la Cour et dont ne s'est pas plaint le 
requérant! Si l'on est en présence d'un recours objectif, d'un préjudice objectif, d'une violation objective de la Charte, il faut que tout cela se termine par une réparation objective. On voit mal par quelle logique juridique l'on devrait compenser par une réparation matérielle ou indemnitaire un préjudice objectif, puisqu'il fallait surtout éviter d'aborder la requête de M. Mtikila comme une action personnelle! En somme, la Cour a semblé faire abstraction de la notion de victime dans l'appréciation du locus standi des requérants, ouvrant la voie devant elle à une logique d'actio popularis, laquelle ne peut manquer de déteindre sur l'examen de la compétence et, surtout, de la recevabilité.

\section{B. L'examen des exceptions d'irrecevabilité par la Cour}

10 Compte tenu de ce que la Cour et la Commission de Banjul fonctionneront en complémentarité sur le terrain de la recevabilité ${ }^{12}$, et au regard de la longue pratique de cette dernière ${ }^{13}$, l'attention devra être vigilante quant à la démarche de la Cour sur le terrain de la recevabilité, non seulement en ce qui concerne l'interprétation de l'article $56^{14}$ de la Charte que les deux institutions interprètent chacune en ce qui la concerne, mais aussi en ce qui concerne la démarche méthodologique suivie par la Cour.

11 La première remarque d'ordre général à faire est que, à ce stade du fonctionnement de la Cour, l'on peine à donner un contenu procédural à la différence entre les articles $38^{15}$, $39^{16}$ et $40^{17} \mathrm{du}$ Règlement de la Cour. En particulier, l'on se demande comment distinguer l'examen préliminaire de la recevabilité, effectué proprio motu par la Cour, et l'examen normal de la recevabilité, une fois que l'affaire a été inscrite au rôle de la Cour.

12 La deuxième remarque porte sur l'ordre d'examen par la Cour des questions de compétence et de recevabilité. Cet ordre est résumé au paragraphe 88 de l'arrêt en ces termes : «les requêtes étant recevables et la compétence en l'espèce étant établie, la Cour procède à l'examen de l'affaire au fond... ». Cette démarche peu habituelle, à en croire le juge Ngoepe dans une "opinion individuelle» elle-même d'une tonalité inhabituelle, divise la Cour. Le juge Ngoepe signale en effet que les arguments en faveur d'un ordre d'examen plutôt qu'un autre sont "solides", même si ces arguments respectifs ne sont pas exposés, ce d'autant plus que selon lui, « le ciel ne s'effondrera pas simplement parce que dans une affaire, la Cour a commencé par traiter de la recevabilité au lieu de la compétence, ou vice versa ». Pourtant, si l'on réserve le cas particulier d'une exception d'irrecevabilité tirée de ce que l'objet de la requête ne rentre pas dans le champ matériel de la compétence de la Cour (article 56-2 de la Charte), exception qui est à la fois une exception d'incompétence matérielle de la Cour et d'irrecevabilité ratione materiae de la requête, la compétence et la recevabilité sont évidemment distinctes et l'examen de la première précède logiquement celui de la seconde. A cet égard, l'on ne peut qu'adhérer à la position de bon sens, de logique et de principe des juges Niyungeko et Ouguergouz exprimée dans leur opinion individuelle respective. Comme le dit en effet l'ancien Président de la Cour, « est-ce que cela aurait un sens qu'il (le juge) commence à s'occuper de ce qu'on lui demande de faire, sans au préalable se préoccuper de savoir s'il peut le faire? La logique et le bon sens commandent que la Cour s'assure d'abord qu'elle a compétence avant d'examiner la requête sous l'angle de la recevabilité ». Pour le juge Ouguergouz, « la Cour doit d'abord 
s'assurer qu'elle a compétence pour connaître d'une requête avant d'examiner la recevabilité de celle-ci ; elle doit le faire proprio motu même lorsque l'Etat défendeur n'a pas soulevé d'exceptions d'incompétence ». Le juge Ngoepe, avons-nous dit, évoque de "solides arguments" en faveur de la thèse opposée. Sauf le respect du secret du délibéré, l'on est curieux d'en prendre connaissance. Car l'enjeu de la question n'est certes pas à la mesure d'un risque d'effondrement du ciel, mais il serait pour le moins cocasse pour la Cour d'avoir à constater qu'elle est incompétente après avoir déclaré une requête recevable ${ }^{18}$. Sans compétence établie, il n'y a pas d'aptitude à trancher la question de la recevabilité.

13 La troisième remarque générale à faire concerne la démarche de la Cour en ce qui concerne l'examen des conditions de recevabilité, démarche qui tranche nettement, dans cette affaire, avec celle suivie par la Commission de Banjul. Selon la jurisprudence établie de cette dernière, les conditions de recevabilité de l'article 56 sont solidaires et doivent, toutes, être examinées à l'occasion d'une affaire, que la partie défenderesse ait ou non soulevé des objections à cet égard. Ainsi, la Commission a-t-elle développé une pratique consistant à examiner des conditions de recevabilité à propos desquelles les parties ne sont pas du tout en désaccord, ou à continuer l'examen de la recevabilité alors même qu'elle avait déjà constaté le non respect d'une condition, ce qui en principe devrait arrêter ledit examen ${ }^{19}$. De même, cette approche a abouti à faire de l'examen de la recevabilité, un stade procédural incontournable et autonome, au terme duquel la Commission doit aboutir à une décision, avant d'entamer le fond de l'affaire. En somme, la recevabilité est examinée de manière systématique, et non dans le cadre d'une procédure incidente destinée à évacuer une exception d'irrecevabilité. A la lecture de l'arrêt de la Cour, l'on se rend compte que cette dernière a limité son examen aux conditions de recevabilité dont le non respect a été soulevé par le défendeur, sans évoquer les autres. Une telle démarche est pertinente et devrait être maintenue par la Cour elle-même et accueillie par la Commission de Banjul. Elle devrait l'être pour au moins deux raisons. D'abord, le procès est l'affaire des parties, et l'organe de contrôle, sauf si le texte adopté par les Etats et l'instituant l'exige, n'a pas à créer des points de divergence que les parties elles-mêmes n'ont pas soulevés. Ensuite, le but de la procédure étant d'examiner une demande au fond relative à des allégations de violation des droits, toute l'énergie procédurale doit être mobilisée à cette fin et n'être contrariée qu'en cas d'incident de procédure exprimé à travers une exception formelle. L'organe de contrôle ne peut prendre sur lui le risque de trouver, par lui-même, un motif d'irrecevabilité non soulevé par le défendeur ${ }^{20}$. Il faut d'ailleurs espérer que les futurs amici curare se garderont d'agir de la sorte.

14 Ces remarques faites, il importe de suivre l'analyse par la Cour des exceptions d'irrecevabilité soulevées, au nombre de deux : le non épuisement des voies de recours internes, le retard dans l'introduction de la requête. L'épuisement des voies de recours internes est la condition de recevabilité la plus examinée devant les mécanismes internationaux de protection des droits de l'homme, et la jurisprudence de la Commission de Banjul est déjà abondante à son sujet ${ }^{21}$. La Cour s'est alignée, pour l'essentiel, sur l'œuvre de la Commission et des autres mécanismes internationaux de garantie, présentée au paragraphe 82.1 comme "la jurisprudence établie». Elle s'appuie sur l'acquis jurisprudentiel concernant la nature des recours concernés, à savoir les recours judiciaires, les caractéristiques de ces recours, à savoir leur disponibilité et efficacité, entre autres. Sur ce point, la Cour n'innove pas. Son alignement sur l'approche de la Commission de Banjul et sur celle de ses devancières 
européenne et interaméricaine vient conforter une situation normative largement stabilisée. Il est du reste à noter que le défendeur lui a particulièrement facilité la tâche, avec cette insistance étrange sur le processus parlementaire qui aurait été en cours sur la question des candidatures indépendantes. La Cour remporte une victoire trop facile, exprimée au paragraphe 82.3 in fine en ces termes: « quelle que soit la nature démocratique du processus parlementaire, celui-ci ne peut pas équivaloir à un processus judiciaire indépendant devant lequel on peut faire valoir des droits consacrés par la Charte ». L'on peut cependant regretter la formule de la Cour contenue dans le même paragraphe selon laquelle "nous constatons que les Requérants ont épuisé les voies de recours internes prévues à l'article 6(2) du Protocole lu conjointement avec l'article 56(5) de la Charte $»^{22}$. Une telle formule est inutilement équivoque, car l'article 6(2) du Protocole ne traite d'aucune voie de recours interne, ni même d'aucune condition de recevabilité et se borne à renvoyer à l'article 56 de la Charte. Il vaut mieux s'en tenir à ce renvoi, en considérant simplement que ce dernier article, en dépit de sa non révision formelle, est aujourd'hui délesté de la référence à la Charte de l'OUA au profit de l'Acte Constitutif de l'Union africaine, comme l'exprime l'article 40 du Règlement de la Cour. ${ }^{23}$

En ce qui concerne le délai d'introduction de la requête, le défendeur estimait qu'il était long et que les requérants devaient être considérés comme forclos. L'argumentation de la Cour sur la question est particulièrement sommaire, alors même que, tout comme sur la question de l'épuisement des voies de recours internes, la «jurisprudence établie » au niveau de la Commission africaine et ailleurs est importante. Sur ce point précis, la Commission de Banjul s'est alignée sur le délai de 6 mois suivant l'acte interne querellé, délai retenu au niveau européen, sauf circonstances spéciales liées au cas sous examen. La Cour, pour des besoins de sécurité juridique, aurait pu reprendre explicitement cette ligne de conduite pertinente. Certes, elle prend en compte les circonstances, puisque son appréciation est effectuée «compte tenu des circonstances » liées à l'attente, par les requérants, de la réaction du Parlement après l'arrêt de la Cour d'Appel confirmant l'interdiction des candidatures indépendantes. L'on retient donc que la Cour statue in concreto. Il est vrai que la circonstance évoquée peut être déroutante, puisqu'il s'agit du processus parlementaire consécutif à l'arrêt de la Cour d'Appel. Or, au moment de l'introduction de la requête, ce processus était, semble-t-il, précisément en cours, sans que l'on sache certes quelles en seraient la durée et l'issue. Si les requérants étaient en droit d' "attendre la réaction du Parlement ", à partir de quel moment pouvait-on estimer que cette attente était libérée parce que la réaction du Parlement tardait à se manifester? La Cour ne se penche pas particulièrement sur cette question.

\section{La question de la compétence ratione temporis de la Cour}

16 Le défendeur estimait que la situation à la base des requêtes, à savoir l'interdiction en 1992 des candidatures indépendantes, était antérieure à l'entrée en vigueur du Protocole de Ouagadougou pour la Tanzanie (soit après le 10 février 2006, date de dépôt de l'instrument de ratification de cet Etat), et à la souscription de la déclaration reconnaissant le droit des individus et des ONG à saisir directement la Cour (29 mars 2010, date du dépôt de la déclaration par la Tanzanie). En conséquence, la Cour ne saurait en connaître. La Cour n'est pas de cet avis, car estime-t- elle, les droits dont la violation est alléguée sont protégés par la Charte de 1981, ratifiée par le défendeur bien avant la survenance des faits litigieux (en 1984). Ces faits se sont prolongés après la 
ratification du Protocole, ainsi qu'après la souscription de la déclaration de l'article 34(6) par la Tanzanie. La Cour se réfère, ce faisant, à la notion de "violation continue " bien connue du contentieux international des droits fondamentaux ${ }^{24}$. L'on pouvait se demander si le recours à l'idée d'allégations de violation qui se prolongent était utile dans ce contexte. En effet, sur la même question de l'interdiction des candidatures indépendantes, il y a eu des actes normatifs, parlementaires ou judiciaires, à différentes périodes de la vie politique de la Tanzanie, et de la vie du droit africain des droits de l'homme dans l'ordre juridique de la Tanzanie. En 1992, il y a eu un acte du Parlement excluant les candidatures indépendantes; en 1994, un acte judiciaire en faveur des candidatures indépendantes; en 1994, un acte du parlement réaffirmant l'interdiction des candidatures indépendantes; en 2006, un acte judiciaire en faveur des candidatures indépendantes ; en 2010, un acte judiciaire contre les candidatures indépendantes. Mais pendant toute cette période, au-delà des actes juridiques, la réalité sur le terrain électoral était celle de l'interdiction des candidatures indépendantes. La compétence de la Cour à l'égard des requêtes individuelles et des ONG visant la Tanzanie débute à partir du dépôt de sa déclaration en vertu de l'article 34(6). Seuls les faits produits à partir de cette date peuvent être déférés à la Cour par ces demandeurs. Les faits antérieurs ne peuvent l'être que si, entamés avant cette date, ils se poursuivent euxmêmes ou dans leurs effets après cette date. Tel est bien le cas de la situation de l'interdiction des candidatures indépendantes. Ayant débuté avant même la naissance de la Cour, elle persiste au moment où la Cour en est régulièrement saisie. Pour la Cour, il ne peut s'agir d'un fait antérieur consommé, mais d'un fait actuel.

17 Les juges Niyungeko et Ouguergouz ont reproché à la Cour, dans leur opinion individuelle respective, de n'avoir pas suffisamment distingué les choses, mêlant les obligations en vertu de la Charte avec celles en vertu du Protocole ou même de la déclaration de l'article 34(6). Pour le premier, «l'on n'aperçoit pas très bien quelle conclusion la Cour tire de la date d'entrée en vigueur de la Charte, par rapport à l'argument de non rétroactivité du Protocole avancé par l'Etat défendeur " (paragraphe 11 de l'opinion). Quant au second, il affirme que «la Cour ne peut être saisie d'allégations de violations des droits de l'homme et des peuples par un individu ou une ONG que si les violations alléguées sont postérieures à l'entrée en vigueur à l'égard de l'Etat concerné non seulement de la Charte africaine mais également du Protocole et surtout de la déclaration facultative» (paragraphe 21 de l'opinion). Cette charge des deux juges peut sembler excessive. On se demande en effet si, même si le propos peut sembler mêlé, ce que le juge Ouguergouz affirme ne se trouve pas au paragraphe 84 de l'arrêt. A notre avis, en substance, la Cour exprime dans ce paragraphe ce que dit, de manière plus savante et professorale assurément, le juge Ouguergouz. Cependant, l'on peut se demander quelle portée il faut accorder au propos du juge Ouguergouz selon lequel « la Cour aurait dû opérer une distinction plus nette entre les obligations de l'Etat défendeur au titre de la Charte africaine et celles qu'il a contractées au titre du Protocole et de la déclaration facultative » (paragraphe 20 de l'opinion individuelle). Techniquement, la Charte, le Protocole et la déclaration sont des instruments juridiques distincts, mais intimement liés, notamment par le fait que le réservoir d'obligations substantielles est dans un instrument unique, la Charte, y compris les textes adoptés ultérieurement pour la compléter ou l'enrichir. Les obligations en vertu de la déclaration se ramènent à l'acceptation d'être cité à comparaître par des individus et ONG, sans plus. Cependant l'objet de la citation n'est pas dans la déclaration, mais plutôt dans la Charte. Par nature, une déclaration de 
l'article 34(6) ne traite pas de questions de substance des droits et tout Etat qui le ferait effectuerait un détournement de finalité de la déclaration ${ }^{25}$. Ensuite, la ratification du Protocole comporte l'obligation de se soumettre aux procédures diligentées devant la Cour par les attributaires du droit de saisine, ainsi que de contribuer au fonctionnement de la Cour. Là encore, cette ratification n'a pas d'effet sur la substance des droits, sous réserve de l'élargissement par l'article 3 du Protocole du droit applicable devant la Cour. En somme, la séparation des obligations ne saurait être exagérée, puisque la Charte reste dans tous les cas le seul réservoir d'obligations substantielles. Les obligations en vertu du Protocole ou de la déclaration de l'article 34(6) ne signifient rien dès lors que l'on fait abstraction de la Charte. Dans le contexte de la complémentarité entre la Commission et la Cour, l'on ne doit pas exclure a priori que la Cour puisse se retrouver en train d'examiner, sur renvoi de la Commission, une affaire concernant un Etat par rapport auquel, suivant l'approche classique de la non rétroactivité, la Cour en principe ne devrait pas exercer sa compétence, parce que probablement elle n'aurait même pas d'effets continus, la violation alléguée ayant été consommée définitivement (meurtre, torture, par exemple).

Quoiqu'il en soit, la Cour devra toujours davantage clarifier son propos. Ce qui est valable au plan de la procédure l'est encore plus lorsque l'on aborde la manière dont la Cour s'est saisie des questions de fond des requêtes.

\section{Une analyse insatisfaisante des questions substantielles soulevées par les requêtes}

Si la Cour africaine doit exercer un leadership technique sur le système africain des droits de l'homme, c'est sur le terrain de l'interprétation substantielle des droits énoncés dans la Charte qu'il lui faudra construire ce positionnement, vis-à-vis de la Commission de Banjul et aussi des juridictions des communautés économiques régionales ${ }^{26}$, lesquelles ont des règles de procédure spécifiques et ne partagent avec les mécanismes dédiés au contrôle international de la Charte que la substance des droits, libertés et devoirs énoncés dans la Charte de $1981^{27}$. A la lecture de l'arrêt du 14 juin 2013, il est clair que si des tendances interprétatives s'affirment nettement, des efforts substantiels sont encore nécessaires pour espérer se hisser, au plan de la démarche technique, au niveau de ses homologues européenne et interaméricaine. Le problème de fond que la Cour avait à adresser est une question de droit constitutionnel partagée par de nombreux Etats parties à la Charte, à savoir la conformité de la prohibition des candidatures indépendantes aux élections et l'obligation symétrique d'être affilié à un parti ou d'être présenté par un parti pour participer aux élections à la Charte africaine des droits de l'homme et des peuples. La République Unie de Tanzanie, comme de très nombreux Etats africains, a retenu depuis 1992 ce système. C'est cette organisation des choses qui est sur la sellette dans les deux requêtes. C'est elle qui est abordée à travers les droits examinés par la Cour, à savoir le droit de participer à la direction des affaires de son pays, la liberté d'association et le droit à l'égalité et à la non-discrimination. La position générale de la Cour est sans équivoque : l'obligation d'affiliation à un parti pour se porter candidat aux élections viole de la même manière tous ces droits. La Cour a repris, à l'occasion de l'examen des requêtes au fond, la démarche jurisprudentielle adoptée par ses homologues européenne ${ }^{28}$ et interaméricaine ${ }^{29}$, et par la Commission africaine. Cette démarche comprend les éléments d'appréciation suivants : nécessité de 
l'ingérence dans une société démocratique, proportionnalité de l'ingérence à l'objectif poursuivi, légitimité du but poursuivi par l'ingérence, limitation des motifs d'ingérence à ceux énoncés à l'article 27(2), construction d'un juste équilibre entre les exigences de l'intérêt général de la communauté et les impératifs de protection des droits individuels fondamentaux. Dans cette démarche la Cour emprunte ${ }^{30}$ massivement aux autres organes de protection, ce qui réduit sa marge de novation et d'autonomie dans l'interprétation. On ne peut que se réjouir de la réception, par la Cour, d'un fond de principes d'examen des ingérences étatiques dans l'exercice des droits qui ont fait leurs preuves au regard de leur haut niveau d'exigence. Pour autant, leur usage massif dans le contexte de la présente affaire laisse un goût mitigé. Pour s'en convaincre, il importe d'examiner successivement les trois droits traités par la Cour.

Auparavant, il est à relever que la Cour a refusé de discuter du moyen tiré de la violation de l'Etat de droit, estimant que « le concept de l'état de droit est un principe d'ensemble dont relèvent tous les droits de l'homme et qui ne saurait être traité dans l'abstrait ou dans la globalité. De plus, l'argument des Requérants selon lequel l'état de droit n'a pas été respecté n'est rattaché à aucun droit spécifique » (paragraphe 121 de l'arrêt). Le refus d'examiner le moyen tiré de la violation de l'Etat de droit peut étonner. D'abord parce que la Cour a retenu la notion de "société démocratique» (paragraphe 106.1) qui ne figure pourtant pas comme telle dans la Charte, notion qui n'est pas en substance éloignée de celle d'Etat de droit; ensuite parce que, en traitant de sa compétence matérielle au paragraphe 85 in fine, "la Cour considère que les violations alléguées relèvent du champ d'application » de l'article 3(1) du Protocole. Or parmi ces «violations alléguées » figure bien la violation de l'Etat de droit. Il n'est pas logique d'affirmer en même temps que les violations alléguées relèvent, toutes, de la compétence matérielle de la Cour, et que l'une d'elles ne peut être traitée par la Cour du fait de sa globalité et parce qu'elle ne cible aucune disposition de la Charte. Il eut été plus simple de ne pas retenir ce moyen, parce que « incompatible avec la Charte " au sens de l'article 56 (2) de ladite Charte. Enfin, le préambule de la Charte renvoie à la Déclaration universelle des droits de l'homme, laquelle, dans le considérant 3 de son propre préambule, énonce qu' "il est essentiel que les droits de l'homme soient protégés par un régime de droit... ", les droits de l'homme étant les choses de l'Etat de droit et de la société démocratique. Le Pr Weckel a salué ce refus d'examiner l'Etat de droit, en avançant «le principe de l'indifférence du droit international à l'égard des questions constitutionnelles $\aleph^{31}$. Ce principe d'indifférence est à relativiser, la jurisprudence internationale des droits de l'homme, en Afrique même, en particulier devant la Commission de Banjul, ayant déjà abordé des questions à impact constitutionnel direct ${ }^{32}$, confirmant ainsi l'idée selon laquelle le droit international des droits de l'homme est un élément au service de la mesure de la qualité de l'ordre constitutionnel, si ce n'est un élément de "l'internationalisation des constitutions " ${ }^{33}$.

21 Cette précision faite, il est temps de revenir à l'examen circonstancié des droits dont la violation est alléguée par les requérants.

\section{A. L'interdiction des candidatures indépendantes au regard du droit de prendre part à la direction des affaires publiques de son pays}

22 La discussion de la Cour sur le droit de prendre part à la direction des affaires publiques de son pays a occupé une part importante de l'examen au fond des requêtes; cette 
discussion a, pour ainsi dire, influencé les conclusions au sujet de la légitimité des ingérences dans les autres droits. Ce droit est consacré à l'article 13(1) de la Charte. Les requérants ayant contesté la légitimité de l'interdiction des candidatures indépendantes, l'Etat tanzanien a estimé que cette interdiction était justifiée par un certain nombre de nécessités liées à la réalité historique et nationale tanzanienne. Au paragraphe 90.1, il est mentionné la nécessité de "garantir la bonne gouvernance et l'unité du pays"; au paragraphe 94, il est fait allusion aux "nécessités sociales du pays » et aux « réalités historiques »; au paragraphe 102, il est évoqué « la structure de l'Union, la République Unie de Tanzanie étant composée de la Tanzanie continentale et de Tanzanie Zanzibar », ainsi que la nécessité « d'éviter tout tribalisme en Tanzanie ». Face à ces arguments, la position de la Cour est nette, telle qu'exprimée au paragraphe 109: «toute loi qui exige du citoyen d'être membre d'un parti politique avant de se présenter aux élections présidentielles, législatives et locales est une mesure inutile, qui porte atteinte au droit du citoyen de participer directement à la vie politique et constitue donc une violation d'un droit ». Il s'agit là d'une condamnation de principe de l'interdiction des candidatures indépendantes, ne découlant nullement de la mise en œuvre de la méthode de recherche du juste équilibre entre les droits individuels et les exigences de l'intérêt général que la Cour a pourtant dit faire sienne, alors même que le défendeur l'y invitait en évoquant l'arrêt de la Cour interaméricaine en l'affaire Castaneda Gutman c. Mexique. Une telle condamnation de principe rend sans objet l'examen des arguments de l'Etat pour justifier la mesure adoptée au plan national. De deux choses l'une : ou bien, en soi, l'interdiction des candidatures indépendantes est une violation de la Charte et il suffit de constater son existence pour conclure à la violation; ou bien il n'en est pas ainsi et l'examen des arguments de l'Etat peut avoir lieu sur le terrain de la légalité, de la nécessité, de la proportionnalité, etc.., et le propos général du paragraphe 109 par conséquent ne mérite pas de figurer dans l'arrêt.

Dès le paragraphe 99 de l'arrêt, et avant même d'avoir mené son analyse, la Cour conclut en ces termes : " vu la clarté manifeste du libellé de l'article 13 (1) de la Charte (...), exiger d'un candidat qu'il soit membre d'un parti politique avant d'être autorisé à participer à la vie politique en Tanzanie constitue certainement une violation des droits consacrés à l'article 13 (1) de la Charte». Nous avons la faiblesse de penser que ce propos, là où il est situé, constitue une faute méthodologique. En concluant avant d'avoir examiné la pertinence des arguments de l'Etat tanzanien justifiant le modèle tanzanien d'aménagement de la candidature à divers types d'élections, la Cour s'est condamnée à motiver à tout prix, à sens unique, dans le sens de la conclusion posée, quitte à faire plus assaut d'amoncellement d'énoncés empruntés çà et là qu'à argumenter in concreto de manière autonome. L'argument de la «clarté manifeste » du droit en cause est une illusion, laquelle a le défaut de faire croire à la Cour qu'elle est dispensée de dire, d'abord, en quoi consiste précisément le droit dont elle constate d'emblée la violation. Or, ce droit ne peut se réduire au droit d'être candidat indépendant à des élections. Les formes de participation à la direction des affaires publiques de son pays sont nombreuses et variables; elles ne sont pas toutes (voire pas du tout) déclinées dans la Charte de $1981^{34}$. Le citoyen peut participer à la direction des affaires publiques en tant qu'électeur, en tant que militant de parti, en tant que candidat, dans les conditions prévues par la loi, ce qui implique qu'en la matière les Etats ont une marge d'appréciation ${ }^{35}$. De quelle étendue ? La Cour n'en dit mot. La Cour ne se penche sur le contenu du droit examiné que treize paragraphes après en avoir affirmé la violation, au paragraphe 107.3, et en recourant à une interprétation extra 
africaine, celle exclusive de l'observation générale du Comité des droits de l'homme $\mathrm{n}^{\circ} 25$, que la Cour fait sienne et considère comme « une déclaration faisant autorité (...), qui reflète l'esprit de l'article 13 de la Charte et qui, en vertu de l'article 60 de la Charte, est 'un instrument adopté par les Nations Unies relatif aux droits de l'homme' dont la Cour peut 's'inspirer' pour sa propre interprétation $»^{36}$. En fait, l'on n'a aucune démarche interprétative, mais l'adhésion à une posture adoptée par une instance quasi juridictionnelle dans un contexte non contentieux. Cette adhésion, dans le contexte de la présente affaire, peut dérouter puisque la Cour affirme au paragraphe 123 qu'il est « inutile» d'examiner l'application, notamment, du Pacte international relatif aux droits civils et politiques dans l'affaire en l'espèce. Finalement, la Cour n'aura pas " appliqué » le Pacte, mais se sera "inspirée » de son interprétation à froid ${ }^{37}$ donnée par le Comité des droits de l'homme pour traiter du moyen tiré du non respect du droit de prendre part à direction des affaires publiques de son pays. D'un autre côté, l'on ne peut se lancer dans un exercice d'examen de la légitimité de ce que l'on appelle rapidement des « restrictions » ou «limitations » d'un droit, sans l'avoir déjà bien défini, et surtout après avoir affirmé sa violation. La violation ne devrait être constatée que si la restriction établie dans la jouissance ou l'exercice d'un droit, au contenu précisé, est dépourvue de légitimité, de nécessité, de proportionnalité, etc. C'est au terme de l'examen des motifs de limitation allégués, au regard de l'ampleur des limitations opérées, que l'on peut, en cas de déséquilibre injustifié au détriment du droit atteint, conclure à la violation. En ayant inversé l'ordre des éléments de l'analyse, la Cour s'est retrouvée obligée d'explorer une somme inutilement intimidante d'espèces jurisprudentielles tirées de partout mais, surtout, d'avoir un discours pro domo, tel que cela apparait aux paragraphes 107.2, 109 in fine, 110 et 111 de l'arrêt.

En tout état de cause, la Cour n'a pas procédé à une analyse rigoureuse de la situation. A notre sens, elle devait d'abord dire ce qu'impose l'article 13 (1) aux Etats et quelle est la marge d'appréciation à cet égard. Ensuite, elle devait dire en quoi l'obligation d'affiliation à un parti par tout potentiel candidat est constitutive, a priori, d'une atteinte au droit garanti par l'article 13 (1). Cela fait, la Cour devait examiner les arguments avancés par l'Etat pour justifier l'orientation de sa réglementation sur le terrain de la légalité, de la nécessité, de la proportionnalité, de manière concrète et contextuelle, et non en général. Ce n'est qu'au terme d'une telle démarche que la Cour pouvait conclure à la violation du droit visé. Or, la Cour commence par affirmer la violation et, seulement ensuite, essaie de l'établir par tous les moyens. Elle commence par traiter longuement, sans raison impérieuse, de l'approche des restrictions aux droits par les divers mécanismes internationaux relatifs aux droits. Après quoi, la Cour examine l'application de cette approche au cas sous examen, si l'on peut dire (car en fait il n'en est rien, les arguments tanzaniens étant plus évacués qu'examinés). Puis la Cour énonce le contenu même du droit dont elle assure la sanction, et achève par des développements généraux sur l'inutilitée ${ }^{38}$ des candidatures patronnées par les partis politiques. Il est évident qu'une telle démarche analytique est tout sauf rigoureuse et intelligible. Le paragraphe 107.2, en particulier, est insatisfaisant, en ce qu'il affirme plus qu'il ne démontre. De plus, il est difficilement compréhensible, lorsqu'il énonce que «les besoins de la population tanzanienne, auxquels sont soumis les droits individuels, doivent, à notre avis être conformes aux obligations individuelles, comme le prévoit l'article 27(2) de la Charte et respecter la sécurité collective, la morale, l'intérêt commun et la solidarité ». Cette phrase est difficilement lisible. Pour la Cour, les restrictions «ne sont pas proportionnelles à l'objectif avancé, qui est le 
renforcement de l'unité et de la solidarité nationale». Naturellement, la Cour ne démontre pas en quoi il y a disproportion par rapport à l'objectif légitime poursuivi. Finalement, de postulats en généralités, la Cour en arrive non seulement à l'affirmation du paragraphe 109 déjà citée, mais aussi aux paragraphes 110 et 111 où la cause dépasse même les requérants pour concerner "les Tanzaniens " dans leur ensemble. A notre sens, l'affirmation du paragraphe 109 est si générale dans sa formulation que l'on peut douter a priori de sa pertinence. Seul le juge Ouguergouz, dans son opinion individuelle, s'est démarqué de cette prise de position de principe de la Cour. Au paragraphe 28 de son opinion individuelle, il écrit: « je considère que l'interdiction des candidatures indépendantes à certaines élections et l'obligation corrélative d'appartenir à un parti politique ne sont pas en elles-mêmes des violations des articles 10 et 13(1) de la Charte africaine; elles ne constituent des violations de ces dispositions que si elles peuvent s'analyser comme des restrictions non raisonnables ou non légitimes à l'exercice des droits consacrés ». Comme la violation de l'article 13(1) a été acquise à l'unanimité, l'on aurait aimé savoir en quoi, pour le juge Ouguergouz, la réglementation tanzanienne était dépourvue de légitimité et de caractère raisonnable.

\section{B. L'interdiction des candidatures indépendantes face à la liberté d'association}

Sur la lancée de ses conclusions relatives au droit de participer à la direction des affaires publiques de son pays, la Cour va aborder la liberté d'association et parvenir à une conclusion identique, à savoir la violation de cette liberté par la Tanzanie. Pour la Cour, «le fait que le Défendeur exige de ses citoyens d'adhérer à un parti politique et d'être investi par celui-ci comme préalable pour se porter candidat aux élections locales, législatives ou présidentielles constitue une entrave à la liberté d'association, puisque les individus sont contraints d'adhérer à une association ou d'en créer une, avant de pouvoir se porter candidat à des postes électifs » (paragraphe 114 de l'arrêt). Pourtant, comme pour le premier moyen, le raisonnement par lequel la Cour parvient à cette conclusion n'est pas convaincant et, une fois de plus, l'on ne peut que marquer de l'étonnement face à l'unanimité des membres de la Cour.

La première remarque à faire est relative à la décision de la Cour d'aborder la question sous l'angle de la liberté d'association tout court. En effet, si les partis politiques sont des formes associatives, il ne s'agit pas d'associations comme les autres, mais des associations politiques, dont la fonction première, depuis l'émergence de ce type organisationnel, est de contribuer à la structuration de l'offre politique, à la formation politique des citoyens, à la formation et à la sélection du personnel politique dirigeant, à l'expression du suffrage. L'histoire politique de l'Afrique, marquée par le phénomène du parti unique, peut justifier une méfiance vis-à-vis de l'obligation d'être patronné par un parti pour être éligible. Mais cela ne saurait autoriser sur le principe une condamnation du rôle des partis dans le jeu politique. Parce qu'ils assument une fonction d'un intérêt public évident pour la régulation de la société politique, les partis politiques, sous certaines conditions, accèdent généralement au financement public de l'Etat. Un parti politique n'est-il qu'une association comme les autres, dont le régime doit être apprécié à l'aune de la liberté générale d'association, sans aucune particularité ? La Cour n'a pas cru devoir se prononcer sur cette question, pourtant importante à notre avis ${ }^{39}$. Elle s'est bornée à rappeler le libellé de l'article 10(2) de la Charte: «nul ne peut être obligé de faire partie d'une association, sous réserve de 
l'obligation de solidarité prévue à l'article 29 ». La Cour reconnaît aux Etats « une certaine marge de discrétion concernant la limitation à la liberté d'association, dans l'intérêt de la sécurité collective, de la morale, de l'intérêt commun et qui respecte les droits et les libertés d'autrui ». Cependant, la Cour ne confronte pas l'interdiction des candidatures indépendantes avec les clauses de limitation relevées, pour apprécier si la Tanzanie a, en l'occurrence, abusé de la «mesure de discrétion" reconnue en la matière aux Etats. Plutôt que cette démonstration circonstanciée, elle énonce une considération de principe : " la Cour estime qu'il ya atteinte à la liberté d'association dès lors qu'un individu est contraint de s'associer avec d'autres personnes. La liberté d'association est aussi bafouée lorsque les autres citoyens sont obligés de s'associer avec un individu. En d'autres termes, la liberté d'association signifie que chacun est libre de s'associer et libre de ne pas le faire" (paragraphe 113). Sans aucune explication, elle décrète au paragraphe 115 ne pas être « convaincue que les nécessités sociales avancées soient conformes aux critères des exceptions prévues à l'article 29 (4) et à l'article 27(2) de la Charte, au point de justifier la limitation du droit du citoyen de choisir de s'associer ou de ne pas s'associer, selon son choix ». Or ce que la Cour devait établir est que, dans le contexte tanzanien concerné, l'intérêt des individus de prendre part à titre individuel à la compétition électorale est parfaitement compatible avec la préservation des réalités sociales, la structure de l'Union, l'unité du pays, etc...; que le besoin social de voir les partis jouer un rôle de premier plan dans le fonctionnement de la société politique tanzanienne, vu ses réalités, peut se concilier avec la volonté des individus de participer à la vie politique et aux élections en dehors des partis politiques. Il n'était pas impossible de l'établir ; il appartenait à la Cour de le faire.

Avec la référence à l'article 29 (4), on est obligé de relativiser la position exprimée par la Cour au paragraphe 107.1 de l'arrêt, où elle «s'accorde avec la Commission africaine pour dire que les limitations aux droits et aux libertés prévues dans la Charte ne peuvent être uniquement que celles qui sont précisées à l'article 27 (2) de la Charte... ». L'examen de la liberté d'association montre clairement que la Cour s'est accordée un peu précipitamment avec la Commission de Banjul, au regard du renvoi explicite de l'article 10(2) de la Charte à l'article 29 de celle-ci, c'est-à-dire à une clause de limitation autre que l'article 27(2).

Dans le cadre de la Charte, le propos suivant lequel « la liberté d'association signifie que chacun est libre de s'associer et libre de ne pas le faire » est excessif, voire erroné en droit, au regard du libellé même de l'article 10 (2) de la Charte. L'obligation de solidarité qui y est mentionnée a précisément pour objet de justifier que l'on puisse contraindre une personne à faire partie d'une association. La contrainte à l'association fondée sur l'obligation de solidarité n'est pas une atteinte à la liberté d'association au regard de la Charte, mais bien plutôt une dimension de cette liberté. La liberté d'association aurait ainsi trois dimensions dans la Charte de 1981: la liberté de s'associer, la liberté de ne pas s'associer, l'obligation de s'associer dans la mesure où l'obligation de solidarité de l'article 29 l'exige, c'est-à-dire lorsqu'il faut " préserver » et «renforcer la solidarité sociale et nationale, singulièrement lorsque celle-ci est menacée ». Ainsi, il y aurait deux niveaux d'appréciation de la légitimité des ingérences étatiques par rapport à la liberté d'association. D'une part, l'article 27(2), clause générale de limitation; d'autre part l'article 29, clause spéciale de limitation. Si la clause générale de limitation est jugée inopérante par l'organe de contrôle, il resterait toujours à apprécier la clause spéciale de limitation. De par l'article 27(2), la liberté d'association (de s'associer ou de ne pas s'associer) doit s'exercer dans le respect, 
notamment, de «l'intérêt commun ». De par l'article 29(4), la liberté d'association dans son volet négatif (ne pas s'associer) peut être contrainte, en vue « de préserver et de renforcer la solidarité sociale et nationale, singulièrement (pas exclusivement, donc) lorsque celle-ci est menacée ». Ce que la Cour avait à établir, pour respecter l'approche en termes de "juste équilibre ", c'est que l'intérêt commun possiblement allégué ne pouvait justifier l'obligation d'affiliation à un parti politique pour prétendre à la candidature à des fonctions électives. Ce que la Cour avait à démontrer, c'est que la préservation et le renforcement de la solidarité sociale et nationale ne pouvaient justifier, en l'occurrence, l'obligation de s'affilier à un parti pour prétendre être candidat à des fonctions de représentation politique au sein de l'Etat. Ces démonstrations, la Cour ne les a pas faites, et sa position est ainsi fragile sur le terrain de l'argumentation juridique. Il ne suffit pas de dire que la Cour n'est pas convaincue des nécessités avancées par l'Etat; encore faut-il démontrer en quoi ces nécessités ne sont pas convaincantes. Faute de le faire, la Cour elle-même ne convainc pas. Dès lors, les affirmations contenues aux paragraphes 113 et 114 n'emportent nullement la conviction sur le terrain de l'interprétation du droit.

\section{L'interdiction des candidatures indépendantes au prisme de la non-discrimination et de l'égalité devant la loi}

Les requérants ont fait valoir que l'interdiction des candidatures indépendantes constituait une atteinte au droit à la non discrimination énoncé à l'article 2 de la Charte, lequel doit être lu en relation avec le droit à l'égalité devant la loi consacré à l'article 3(2) de la Charte. Au paragraphe 116 de l'arrêt, il est dit qu'ils soutiennent que les dispositions constitutionnelles ont pour effet de créer une discrimination à l'égard de la majorité des Tanzaniens. Au paragraphe 117, cet argument est étayé par celui en vertu duquel «seuls ceux qui sont membres d'un parti politique et qui sont parrainés par celui-ci peuvent se présenter aux élections présidentielles, parlementaires ou locales ", les autres en étant exclus. Il y a donc une "différence de traitement entre Tanzaniens» du fait des dispositions constitutionnelles querellées. Le propos est confus. Tantôt, les requérants affirment que la discrimination est un «effet » des dispositions constitutionnelles (paragraphes 116 et 119), tantôt, en citant au paragraphe 117 in fine la décision de la Commission de Banjul en l'affaire Legal Resource Foundation c. Zambie, que les dispositions constitutionnelles visaient à (et donc avaient pour but de) exclure certains citoyens du processus démocratique, et finalement au paragraphe 119 que la différence de traitement était «inscrite dans les amendements constitutionnels ». La Cour partage l'argumentation des requérants sur l'existence d'une différence de traitement, et sur le fait qu'une telle différence ne peut être justifiée par les nécessités invoquées par l'Etat.

30 La Cour estime au paragraphe 119 que «à la lumière de l'article 2 de la Charte(...), la discrimination alléguée pourrait être apparentée à une distinction basée sur une 'opinion politique ou ...toute autre opinion' ». Ce propos visiblement peu élaboré laisse dubitatif. On ne perçoit pas clairement ce que la Cour considère comme une " opinion » sur le fondement de laquelle il y aurait eu une attitude discriminatoire de la part des autorités tanzaniennes. Est-ce le fait de contester l'interdiction des candidatures indépendantes ? Si telle est l' "opinion » retenue par la Cour, il est à relever que la réglementation tanzanienne n'exclut pas de la candidature les contestataires de l'obligation d'affiliation partisane. Du reste, quoique contempteur déclaré d'une telle 
obligation, M. Mtikila a été admis à prendre part aux élections dans son pays en tant que candidat, sans que l'expression de son opinion, en tant que telle, lui ait valu l'exclusion de la compétition électorale. Il n'a été démontré, ni par les requérants, ni par la Cour, que les dispositions constitutionnelles tanzaniennes avaient pour but affiché ou caché, au moment de leur élaboration, d'exclure une certaine opinion politique.

31 En tout état de cause, le raisonnement de la Cour sur la non discrimination est, à notre avis, faible. En effet, il n'est pas expliqué pourquoi la distinction à examiner sur le terrain de la non- discrimination est celle qui oppose les citoyens « membres d'un parti politique " et les citoyens "non membres d'un parti politique». A notre sens, la distinction pertinente n'est pas celle-là. La distinction à prendre en compte est celle qui existe entre les Tanzaniens qui veulent se présenter comme candidats aux élections et ceux qui, quoique participant également à la vie politique de leur pays en tant que citoyens, n'entendent pas le faire dans la posture de candidats. La citoyenneté ne se réduit pas à l'éligibilité; cette dernière est une situation spécifique par rapport à laquelle l'on peut examiner la légitimité d'éventuelles différences de traitement. Or, si l'on considère que la situation comparable de base n'est pas le statut général de citoyen, lequel est d'un spectre large, mais le statut de candidat, lequel ne concerne qu'une catégorie circonscrite de citoyens, l'on doit noter que tous les citoyens tanzaniens, absolument tous, sans distinction, désireux d'être candidats aux divers types d'élections, sont soumis aux mêmes règles régissant la candidature, y compris l'obligation d'être affilié à un parti politique. De telles règles, faut-il le rappeler, ne peuvent être définies de manière exhaustive par un instrument international ou même un organe international compétent en matière de droits fondamentaux, la marge d'appréciation des Etats étant, dans un domaine où les considérations d'opportunité et autres sont inévitables, incompressible ${ }^{40}$. Il y aurait une distinction de caractère défavorable, une discrimination, si, parmi les citoyens désireux d'être candidats, certains étaient libérés par la loi ou par la pratique, pour des motifs illégitimes, de l'obligation d'affiliation à un parti, alors que d'autres y étaient astreints. Au regard des faits de l'affaire tels qu'ils ont été reproduits dans l'arrêt, l'on voit difficilement comment, en dehors de cette approche, la Cour pouvait établir un comportement discriminatoire de la part de la Tanzanie.

\section{Conclusion}

32 La Cour d'Arusha a conclu son premier arrêt au fond sur une condamnation de l'Etat tanzanien. La réparation quant à elle pourrait intervenir ultérieurement. On peut néanmoins se demander si la Cour a le droit, comme elle l'a fait au paragraphe 124 in fine de son arrêt, d'inviter un requérant, si ce denier le souhaite, à demander une compensation ou une réparation. Au regard de la lettre des articles 27(1) du Protocole et $63 \mathrm{du}$ Règlement intérieur de la Cour, l'on est en droit d'en douter. Le premier article autorise la Cour, de son propre chef, à décider d'une compensation ou d'une réparation lorsqu'elle constate une violation, ce qu'elle n'a pas fait alors que les premiers requérants le lui avaient explicitement demandé. Le second article prévoit que la Cour statue sur une demande de réparation introduite par le requérant, ce que le requérant n'a pas fait tout au long de la procédure, alors même qu'il s'était réservé le droit " de compléter l'analyse juridique » sur la question dans sa requête introductive d'instance. Il est clair, à notre sens, que le droit réservé devait s'exercer avant le délibéré et ne 
devait pas être mis en mouvement seulement après le prononcé de l'arrêt constatant la violation, dans un tête à tête processuel entre le demandeur et la Cour, à l'exclusion du défendeur. La circonstance que la Cour puisse se prononcer sur la réparation « dans un arrêt séparé ", distinct de l'arrêt sur le principal "si les circonstances l'exigent ", n'implique pas que la demande de réparation doive être présentée en dehors et après la procédure sur le principal. La demande de réparation, détaillée, doit faire partie des conclusions du demandeur communiquées au défendeur pendant le procès. $\mathrm{Si}$, selon l'article 34 (5) du Règlement de la Cour, le principe de la demande de réparation doit figurer dans la requête initiale, le montant de celle-ci et les éléments de preuve y relatifs pouvant être soumis "ultérieurement ", ce stade ultérieur ne peut aller au-delà du délibéré et du prononcé de l'arrêt sur le principal. La distinction possible des arrêts sur le principal et sur la réparation n'implique nullement une autonomisation des procédures sur le fond et sur la réparation. Une approche différente, soit ouvrant un nouveau procès, soit organisant une procédure en dehors du défendeur, heurterait à notre sens le principe de bonne administration de la justice.

Cela dit, loin de nous la prétention d'épuiser la richesse du premier arrêt au fond de la Cour africaine des droits de l'homme. Il montre le courage d'une jeune juridiction qui n'hésite pas à aborder des questions sensibles de droit constitutionnel, voire de pratique politique, avec leurs implications sur le terrain des modalités de compétition pour le pouvoir et de représentation politique au sein des Etats. Ce faisant, comme la Commission de Banjul, la Cour se positionne comme l'un des postes avancés de l'accompagnement des Etats africains vers la consolidation de leur ouverture démocratique et vers le renforcement de pratiques constitutionnelles et politiques respectueuses des droits fondamentaux, des principes démocratiques ${ }^{41}$. C'est pourquoi, avec la perspective réelle d'accueillir des allégations de violation de la Charte africaine de la démocratie, des élections et de la gouvernance ${ }^{42}$, la Cour devrait éviter de refuser purement et simplement d'aborder le moyen tiré de la violation de l'Etat de droit. L'Etat de droit est un concept dont les éléments structurants sont suffisamment identifiables et dont les interactions avec les droits fondamentaux sont si évidentes qu'il importe que la Cour, méthodiquement bien entendu, s'en saisisse à bras le corps, pour aider les jeunes démocraties africaines sur le vaste chantier de la construction de sociétés politiques humaines, décentes, conviviales, épanouissantes pour tous. Naturellement, il lui faudra faire preuve de prudence tactique, en évitant des postures de principe trop générales et catégoriques, et en retenant une approche contextuelle du juste équilibre entre les nécessités sociales et les droits fondamentaux de l'individu. La Cour ne doit pas se plier au contexte africain des droits de l'homme, souvent dangereux pour les droits, mais doit se positionner de manière à en accompagner résolument, mais méthodiquement, la subversion. Dans cette démarche faite de résolution ferme et de prudence méthodique, la Cour doit pouvoir résister aux pressions de la communauté des ONG des droits de l'homme, laquelle pourrait l'acculer à un maximalisme interprétatif incompatible avec la prudence nécessaire attendue $\mathrm{du}$ juge, y compris le juge préposé à la garantie des droits de l'homme. Le dynamisme interprétatif doit refléter aussi, autant que possible, la réalité des évolutions concrètes de la garantie des droits en Afrique. Rien ne serait plus abstrait que d'avoir à Banjul et à Arusha une jurisprudence futuriste, supportant la comparaison technique avec celle des autres mécanismes internationaux, mais avec une réalité timide des progrès des droits de l'homme sur le continent. Les amateurs de belles formules techniques auraient matière à s'en donner à cœur joie et à remplir les revues de commentaires 
savants, mais tout cela serait comme une machine tournant à vide. A cet égard, l'on ne peut s'empêcher d'exprimer quelque amusement gêné devant le rappel, par des protagonistes de l'affaire, des circonstances dans lesquelles tout cela a été déclenché, comment M. Mtikila a été presque sollicité pour voir la Cour rendre un premier arrêt au fond ${ }^{43}$. Si les Etats, encore frileux dans leur grande majorité à souscrire la déclaration de l'article 34(6) de la Charte, venaient à être convaincus que la Cour pourrait se transformer en une arène où l'on vient effectuer des jeux de droit, ce serait un coup dur pour l'avenir du recours individuel devant la juridiction africaine exclusivement dédiée à la garantie des droits de l'homme sur le continent. Nous n'en sommes pas là, heureusement, et il faut espérer que de tels augures ne se réaliseront pas.

\section{NOTES}

1. Olinga A. D., «L'émergence progressive d'un système africain de garantie des droits de l'home et des peuples ». in Olinga A. D. (dir.), La protection internationale des droits de l'homme en Afrique. Dynamique, enjeux et perspectives trente ans après l'adoption de la Charte africaine des droits de l'homme et des peuples. Yaoundé, Editions Clé, 2012, pp. 13-35.

2. Voir état des affaires devant la Cour africaine in http://www.african-court.org/fr/index.php. 19 février 2014. Sur la première décision de la Cour, voir Olinga A.D. « Regards sur le premier arrêt de la Cour africaine des droits de l'homme ». RTDH, $n^{\circ} 83,2010$, pp.749-768. Voir également Kilangi A. "Legal personality, responsibility and immunity of the African Union: Reflection on the decision of the African Court on Human and peoples' Rights in the Femi Falana case", AUCIL Journal of International Law, vol. 1, 2013, pp. 95-139 ; voir aussi Koagne Zouapet Apollin. " Une si évidente décision...une cour bien confuse : quelques observations sur l'arrêt de la Cour africaine des droits de l'homme et des peuples en l'affaire Femi Falana c. Union Africaine (affaire n001/2011) ». in www.africancourtcoalition.org Août 2012.

3. Au 31 décembre 2013, sept Etats avaient souscrit la déclaration de l'article 34(6) de la Charte, à savoir : le Burkina Faso, la Côte d'Ivoire, le Ghana, le Malawi, le Mali, le Rwanda, la Tanzanie.

4. Voir Lekene Donfack E.C., «La candidature indépendante et la liberté de suffrage en droit camerounais ", Revue Africaine des Sciences Juridiques, 1 (2000), pp. 21-52.

5. Observation Générale $\mathrm{n}^{\circ} 25$ du Comité adoptée lors de sa 57è session, le 12 juillet 1996, Participation aux affaires publiques et droit de vote. Texte disponible in HRI/GEN/1/Rev.9 (Vol. I), 27 mai 2008, pp. 255-260.

6. Notamment l'arrêt de la Cour en l'affaire Castaneda Gutman c. Mexique, 6 août 2008.

7. Voir Santulli C., Droit du contentieux international. Paris, Montchrestien/Domat, 2005.

8. Voir Olinga A.D., « Pratique de la Cour africaine des droits de l'homme et des peuples en 2011 », RTDH, n93, $1^{\text {er }}$ janvier 2013, pp. 123-142.

9. Voir Olinga A.D., « Vers un contentieux objectif à Banjul ? L'affaire Lawyers for Human Rights contre Royaume du Swaziland devant la Commission africaine des droits de l'home et des peuples ", Revue Juridique et Politique des Etats Francophones, 2007, 1, pp. 28-52.

10. Voir, sur la victime, Tulkens F., « Victimes et droits de l'homme dans la jurisprudence de la Cour européenne des droits de l'homme ", in Archives de Politique Criminelle, 2002/1, n²4, pp. 41-59. Pour une variation synthétique récente sur cette question, voir Renucci J.-F., « La notion 
de 'victime' au sens de l'article 34 de la Convention européenne des droits de l'homme ", Recueil Dalloz, 2014, pp. 38 et s.

11. Le paragraphe 74 de l'arrêt renseigne que c'est au lendemain de l'arrêt la Cour d'appel $\mathrm{qu}^{\prime}$ « un processus de consultation visant à recueillir l'opinion des citoyens Tanzaniens sur une éventuelle modification de la Constitution " a été lancé, processus en cours au moment de la saisine de la Cour, et probablement au moment du prononcé de l'arrêt, lequel serait venu interférer dans un processus politique interne.

12. Voir Ebobrah Solomon T., " Towards a positive application of complementarity in the African Human Rights System: issues of functions and relations", EJIL (2011), vol.22, n³, pp.663-688.

13. Ayina Ayissi F. \& Zanga M.J. «La procédure d'examen des communications devant la Commission de Banjul ». in Olinga A.D. (dir.), La protection internationale des droits de l'homme en Afrique. Dynamique, enjeux et perspectives trente ans après l'adoption de la Charte africaine des droits de l'homme et des peuples. Yaoundé, 2012, pp. 151-189.

14. Voir Ouguergouz F . «Article 56 ", in Kamto M. (Dir.), La Charte africaine des droits de l'homme et des peuples et le Protocole y relatif portant création de la Cour africaine des droits de l'homme et des peuples. Commentaire article par article», Bruxelles, Editions Bruylant, Editions de l'Université de Bruxelles, 2011.

15. Rejet d'une requête manifestement mal fondée.

16. Examen préliminaire de la compétence de la Cour et de la recevabilité de la requête.

17. Conditions de recevabilité des requêtes.

18. Il faut relever pourtant, pour le déplorer, que la Cour persiste dans son approche, avec une situation particulièrement chaotique dans l'arrêt de la Cour en l'affaire Urban Mkandawire c. Malawi en date du 21 juin 2013. Ici, la Cour examine d'abord une exception d'incompétence ratione temporis (paragraphe 32), puis évacue l'exception d'irrecevabilité liée à la saisine par le requérant d'un autre mécanisme international de règlement (paragraphe 33), puis revient aux aspects de la compétence, à savoir les compétences ratione materiea, ratione personae et encore ratione temporis (paragraphes 34,35 et 36 ), et conclue par l'examen particulier de la condition relative à l'épuisement des voies de recours internes (paragraphes 38 à 40.2). Comme l'ont à juste titre relevé les juges Guissé et Niyungeko dans leur opinion dissidente commune, cette démarche est confuse et manque de cohérence.

19. Voir sur cette question Olinga A.D. «Remarques introductives sur l'interprétation de la Charte africaine des droits de l'home par la Commission de Banjul ", in Olinga A.D. (Dir.) La protection internationale des droits de l'homme en Afrique. Dynamique, enjeux et perspectives trente ans après l'adoption de la Charte africaine des droits de l'homme et des peuples. Yaoundé, Editions Clé, 2012, pp. 219-248.

20. Malheureusement, il faut se rendre à l'évidence que la Cour ne s'en est pas tenue à cette approche pertinente bien longtemps, puisque au cours du même mois de juin 2013, dans l'affaire Urban Mkandawire c. Malawi, la Cour va précisément aboutir à ce résultat invraisemblable que l'on redoutait, à savoir prononcer l'irrecevabilité d'une requête sur la base du non respect par le requérant d'une condition de recevabilité dont le non respect n'a pas été soulevé par l'Etat défendeur, en l'occurrence la condition d'épuisement des voies de recours internes. Dès le paragraphe 33, la Cour estime que l'exception de litispendance est non fondée, mais ajoute tout de suite que « ce constat ne signifie pas nécessairement que la requête est recevable, car elle doit encore remplir d'autres critères de recevabilité en particulier le Requérant doit satisfaire aux dispositions de l'article 6(2) du Protocole, lu conjointement avec l'article 56(5) de la Charte et démontrer qu'il a épuisé les voies de recours internes ». C'est au paragraphe 38 de l'arrêt que la Cour se livre à cet examen : «le Défendeur n'a pas soulevé d'exception de non -épuisement des recours internes. La Cour a cependant le devoir de faire respecter les dispositions du Protocole et de la Charte. Elle est tenue de s'assurer que la requête est conforme, entre autres, aux conditions de recevabilité énoncées dans le Protocole et dans la Charte. La loi ne doit pas faire débat. Le fait 
pour le Défendeur de ne pas soulever la question de la non-conformité avec les exigences inscrites dans le Protocole et la Charte ne peut pas rendre recevable une requête qui est autrement irrecevable. L'épuisement des recours internes est une règle fondamentale dans la relation entre les Etats Parties avec le Protocole et la Charte et avec les juridictions nationales d'une part, et avec la Cour, d'autre part. Les Etats Parties ratifient le Protocole en tenant pour acquis que les recours internes doivent d'abord être épuisés avant que la Cour ne soit saisie ; la déclaration prévue à l'article 34(6) du Protocole est également faite sur cette base ». Ce propos est d'une portée procédurale considérable. Il signifie tout d'abord que la Cour s'aligne finalement sur la doctrine de la Commission d'examen des conditions de recevabilité au-delà des exceptions soulevées explicitement par le défendeur. Pour autant, cet examen ne semble pas nécessairement systématique, puisque la Cour, en ne traitant que de la condition de l'épuisement des voies de recours internes, et non des autres conditions, semble considérer cette dernière comme celle dont l'examen s'impose en tout état de cause à l'organe de contrôle, parce qu'elle participerait de l'esprit même de subsidiarité dans lequel a été conçue la mécanique internationale de garantie, esprit qui serait le fondement même de l'adhésion des Etats à cette mécanique. Finalement, audelà de la perspective de l'examen systématique des conditions de recevabilité, la Cour vient de consacrer pour la condition d'épuisement de voies de recours internes, le statut d'une condition impérative, dont la Cour doit "s'assurer du respect ", que l'Etat défendeur soulève ou non d'exception à cet égard. Avec cette évolution, le système africain se signale comme le seul où un organe international de contrôle peut prendre sur lui de déclarer irrecevable une requête, sur la base du non respect, découvert par ledit organe, d'une condition de recevabilité dont le respect n'a pas été contesté par le défendeur, une condition qui devient dès lors une condition d'ordre public. Une telle évolution des choses est inattendue et déconcertante.

21. Pour une étude riche et particulièrement bien menée, voir Nsogurua J. Udombana, «"So far, so fair: the local remedies in the jurisprudence of the African Commission on Human and Peoples' Rights", in The American Journal of International Law, vol. 7, 1, 2003, pp. 1-37.

22. Nos italiques.

23. Il est vrai qu'en l'absence de révision formelle de la Charte de 1981, pour la délester de toutes les références à la Charte de l'OUA, on peut se montrer dubitatif, au plan purement technique, sur la substitution automatique de l'Acte constitutif de l'UA à la Charte de l'OUA. Certes, l'un et l'autre instrument sont à la base de la mise en place de l'organisation continentale au sein de laquelle est créée la Cour ; toutefois, le contenu substantiel de ces deux instruments n'est pas identique. L'Acte Constitutif de l'UA est incontestablement plus fourni en matière de principes et de règles relatives aux droits de l'homme que la charte de l'OUA.

24. Voir Sudre F., Droit international et européen des droits de l'homme, Paris, PUF, 5è édition, 2001, p. 420 .

25. On peut se demander, à cet égard, quelle portée devrait être accordée, sur le terrain de la détermination de la compétence matérielle de la Cour, au renvoi à la Constitution de la République de Tanzanie opéré dans la déclaration de ce pays en vertu de l'article 34(6), de manière générale et, spécifiquement, dans le contexte de l'affaire sous examen. Si ce renvoi signifie que les plaignants en vertu des articles 5(3) et 34(6) du Protocole ne peuvent introduire contre la Tanzanie que des requêtes compatibles, non seulement avec l'Acte constitutif de l'Union africaine et la Charte de 1981, mais aussi avec la Constitution de la Tanzanie, alors il faudrait simplement dire que les requêtes ayant conduit à l'arrêt, mettant directement en cause une clause de la Constitution tanzanienne, ne pouvait être « in adherence to the Constitution of the United Republic of Tanzania » selon les termes de la déclaration du 29 mars 2010.

26. Voir Djoumessi Kenfack S. B., "L'application de la Charte africaine des droits de l'homme et des peuples par les juridictions des Communautés économiques régionales ", in Olinga A.D. (dir.), La protection internationale des droits de l'homme en Afrique. Dynamique, enjeux et perspectives trente 
ans après l'adoption de la Charte africaine des droits de l'homme et des peuples. Yaoundé, Editions Clé, 2012, pp. 251-279.

27. Voir Adjolohoun Horace S. «The ECOWAS Court as human rights promoter? Assessing five years' impact of the Koraou Slavery judgment ». in Netherlands Quarterly of Human Rights, vol. 31/3, 2013, pp. 342-371; voir aussi Karen J. Alter, Laurence R. Helfer \& Jacqueline R. Mc Allister, “ A new international human rights court for West Africa: the ECOWAS Community Court of Justice". The American Journal of International Law, 2013, vol.107, pp. 737 et s.

28. Ost F., «Originalité des méthodes d'interprétation de la Cour européenne des droits de l'homme », in Delmas-Marty M. Raisonner la raison d'Etat. Paris, PUF, 1989, pp. 405-463.

29. Burgorgue-Larsen Laurence, «Les méthodes d'interprétation de la Cour interaméricaine des droits de l'homme. Justice in context », RTDH, n 97/2014, pp. 23-71.

30. Voir Olinga A.D., "Les emprunts normatifs de la Commission africaine des droits de l'homme et des peuples aux systèmes européen et interaméricain de garantie des droits de l'homme ", RTDH, n 62/2005, pp. 501-537.

31. Weckel Ph. «Présentation ». in Adjovi R. (Dir.) Cour africaine des droits de l'homme et des peuples. L'affaire Rev. Mtikila c. Tanzanie. Dossier du Pôle Afrique de Sentinelle. http://www.sentinelle-droitinternational.fr., 02 mars 2013, p. 3.

32. Voir, notamment, les affaires suivantes : Legal Resource Foundation c. Zambie, 2001 ; Lawyers for Human Rights c. Swaziland, 2005 ; Mouvement Ivoirien des droits humains c. Côte d'Ivoire ; John K. Modise c. Botswana, 6 novembre 2000; Zimbabwe Lawyers for Human rights and Associated Newspapers of Zimbabwe c. Zimbabwe; Kevin Mgwanga Gumne et al. C. Cameroun, 2009. Les juridictions sous régionales ne sont pas en reste, comme le montre l'arrêt de la Cour de justice de la CEDEAO en l'affaire Aneganvi Manavi Isabelle et autres $c$. Togo du 7 octobre 2011, arrêt qui met sur la sellette un problème de mandat impératif, question constitutionnelle s'il en est. La Cour de la CEDEAO a condamné l'Etat du Togo dans la cause.

33. Voir Ondoua Alain, «L'internationalisation des Constitutions en Afrique subsaharienne francophone et la protection des droits fondamentaux ", RTDH, $n^{\circ} 98$, pp.437-457. L'auteur relève, en page 455, que la condamnation de la Tanzanie par la Cour « est prononcée alors même que la Cour d'appel de Tanzanie n'y avait décelé aucune inconventionnalité ». Sauf erreur de notre part, il ne semble pas que le moyen tiré de la violation de dispositions conventionnelles ait été jamais soulevé devant les juridictions tanzaniennes par M. Mtikila, le débat ayant été mené par rapport aux principes gouvernant l'Etat de droit, dans le cadre de l'ordre juridique et politique de la Tanzanie. Par deux fois devant la Haute Cour, en 1993 puis en 2005, M. Mtikila a toujours fait valoir des arguments relatifs à la non-conformité de l'interdiction des candidatures indépendantes à la Constitution tanzanienne. Du reste, dans sa décision du 5 mai 2006, près de douze ans après la première rendue sur la même question le 24 octobre 1994, la Haute Cour estime que « les modifications contestées violaient les principes démocratiques et la doctrine des structures fondamentales inscrites dans la Constitution» (paragraphe 72 de l'arrêt).

34. Parce que ces obligations ne sont pas déclinées dans la Charte, la référence à l'article 27 de la Convention de Vienne de 1969 (paragraphe 108) ou à l'article 32 du Projet d'articles de la Commission du Droit International sur la responsabilité internationale des Etats n'est pas pertinente. Lorsque l'étendue de l'obligation est liée à la marge nationale d'appréciation, lorsque le droit interne est invité à participer à la circonscription du champ de l'obligation elle-même, les notions de "non exécution du traité » et de "manquement aux obligations », sans disparaître, sont à relativiser.

35. Voir Kovler Anatoly, «La Cour européenne des droits de l'homme face à la souveraineté d'Etat ", in L'Europe en formation, 2013/2, n 368, pp. 209-222.

36. La qualification attribuée à l'observation générale peut étonner. De toute évidence, il ne s'agit pas à notre avis d'un « instrument adopté par les Nations Unies relatif aux droits de l'homme ». 
37. Sur le «flottement entre interprétation et application » lié au terme "inspiration », voir Decaux E., « Article 60 ». in Kamto M. (dir.), La Charte africaine des droits de l'homme et des peuples et le Protocole y relatif portant création de la Cour africaine des droits de l'homme et des peuples. Commentaire article par article ». Bruxelles, Editions Bruylant, Editions de l'Université de Bruxelles, 2011, notamment p. 1112.

38. L'on se demande s'il appartient à un mécanisme international de garantie de se prononcer sur l'utilité des mesures nationales. L'intrusion dans ce domaine peut confiner à un contrôle d'opportunité, lequel en principe ne relève pas de la compétence des instances internationales.

39. Voir Tajadura Tejada Javier, «La doctrine de la Cour européenne des droits de l'homme sur l'interdiction des partis politiques ", RFDC, 2012, 2, n 90, pp. 339-371.

40. Voir Levinet Michel, «Droit constitutionnel et Convention européenne des droits de l'homme. La confirmation de l'autonomie des Etats en matière de choix des systèmes électoraux. Brèves réflexions sur l'arrêt rendu par le Cour européenne des droits de l'homme dans l'affaire Yumak et Sadak c. Turquie (Gr. Ch., 8 juillet 2008) ", RDFC, n 78, 2009, pp. 423-430.

41. Olinga A.D. «L'impératif démocratique dans l'ordre régional africain », Revue de la Commission africaine des droits de l'homme et des peuples, vol. 6, n 2, 1998, pp. 57-80.

42. Djpoumessi Kenfack S. B., La Charte africaine de la démocratie, des élections et de la gouvernance à l'épreuve des changements anticonstitutionnels de gouvernement, Mémoire, Master II en Contentieux International, IRIC, Yaoundé, 2012, 187 p.

43. Lire à cet égard Adjovi R. «Historique de l'affaire Rév. Mtikila c. Tanzanie». in Adjovi, R. (dir.), Cour africaine des droits de l'homme et des peuples. L'affaire Ré. Mtikila c. Tanzanie, op. cit., pp. 5-7.

\section{ABSTRACTS}

With the Reverend Mtikila vs Tanzania case, the African Court on Human and People's Rights isued it first decision on merits. Apart from issues related to procedure, about which the members of the Court did not agree, the decision is an instructive one, as it deals with the conformity to the African Charter of the obligation to be affiliated to a political party for taking part to elections. In the view of the Court, such an obligation violates the right to participate in the government of his country, the right to assemble with others, the right to be protected from discrimination. The Court finds that the principle of the rule of law is not relevant. Although the reasoning of the court can be difficult to follow, the decision is an important step for its mission in favour of human rights in Africa.

La Cour africaine des droits de l'homme et des peuples a rendu, en l'affaire Révérend Mtikila c/ Tanzanie, son premier arrêt au fond. Au-delà de certains aspects de procédure sur lesquels les juges ont eu quelque difficulté à s'accorder, la décision est instructive, car elle traite de la conformité de l'obligation d'affiliation partisane pour tout candidat aux élections avec la Charte africaine des droits de l'homme et des peuples. Pour la Cour, une telle obligation viole le droit de participer à la direction des affaires publiques de son pays, la liberté d'association et le droit à la non-discrimination. En revanche, l'atteinte au principe de l'Etat de droit n'est pas retenue. Si l'on peut avoir du mal à suivre la logique argumentative de la Cour, il reste que cet arrêt constitue une contribution importante à l'œuvre inaugurale de cette juridiction en faveur des droits de l'homme en Afrique. 
INDEX

Mots-clés: Compétence - Recevabilité - Locus standi - Restrictions - Droit de participer à la gestion des affaires publiques - Liberté d'association - Non-discrimination - Partis politiques Etat de droit

Keywords: Competence - Recevability - Locus standi - Restrictions - Right of participation to the public policies - Freedom of association - Non-discrimination - Political parties - Rule of law

\section{AUTHOR}

\section{ALAIN DIDIER OLINGA}

Alain Didier Olinga, Docteur/HDR en Droit Public de l'Université de Montpellier I, est Maître de Conférences à l'Université de Yaoundé II (Cameroun). Il est chef du Département de Droit International à l'Institut des Relations Internationales du Cameroun (IRIC), au sein de cette Université. 